\title{
THE
}

$12-1988$

\section{Regular and Chaotic Time Evolution in Spin Clusters}

Niraj Srivastava

Charles Kaufman

University of Rhode Island, ckaufman@uri.edu

Gerhard Müller

University of Rhode Island, gmuller@uri.edu

Follow this and additional works at: https://digitalcommons.uri.edu/phys_facpubs

Terms of Use

All rights reserved under copyright.

\section{Citation/Publisher Attribution}

Niraj Srivastava, Charles Kaufman and Gerhard Müller. Regular and chaotic time evolution in spin clusters. J. Physique C8 (1988), 1601-1602.

Available at: http://dx.doi.org/10.1051/jphyscol:19888733

This Article is brought to you for free and open access by the Physics at DigitalCommons@URI. It has been accepted for inclusion in Physics Faculty Publications by an authorized administrator of DigitalCommons@URI. For more information, please contact digitalcommons-group@uri.edu. 


\title{
Regular and Chaotic Time Evolution in Spin Clusters
}

\author{
Niraj Srivastava, Charles Kaufman and Gerhard Müller \\ Department of Physics, University of Rhode Island, Kingston RI 02881, USA
}

\begin{abstract}
We calculate spin-autocorrelation functions (as time averages over chaotic trajectories) and their intensity spectra for clusters of two classical spins, interacting via a nonintegrable Hamiltonian. The long-time behavior observed includes both power-law decay and persistent oscillatory components, resulting in an intensity spectrum with power-law singularities and discrete lines.
\end{abstract}

The dynamics of clusters of two or more classical spins, vectors of fixed length which interact via a prescribed Hamiltonian, is a powerful yet convenient way to investigate the nature of classical chaos and its possible manifestations in the corresponding quantum systems [1-5]. The convenience is due to the relative straightforwardness of the technical problem; the method is powerful because the phase space is compact and the energy strictly bounded, and so truncations or approximations, which might obscure the essence of the problem, can be avoided. In previous work [3-5] we have studied the nature of the trajectories for various Hamiltonians, their geometric structure, as well as the existence and analytic nature of invariants of the classical motion. We are continuing our study of these systems, and report here new results on time correlation functions and spectral properties of classical 2-spin clusters.

We consider the Hamiltonian

$$
H=\sum_{\alpha=x, y, z}\left\{-J_{\alpha} S_{1}^{\alpha} S_{2}^{\alpha}+\frac{1}{2} A_{\alpha}\left[\left(S_{1}^{\alpha}\right)^{2}+\left(S_{2}^{\alpha}\right)^{2}\right]\right\} .
$$

The time evolution of the spin vectors Sf is determined by Hamilton's equation,

$$
\mathrm{d} \mathbf{S}_{\ell} / \mathrm{d} t=-\mathbf{S}_{\ell} \times \partial H / \partial \mathbf{S}_{\ell}=\left\{H, \mathbf{S}_{\ell}\right\}, \quad \ell=1,2 .
$$

That the variables $\mathbf{S}_{\ell}$ correspond to spins is guaranteed by the requirement that the Poisson brackets of their components satisfy the commutation rules of angular momentum variables,

$$
\left\{S_{\ell}^{\alpha}, S_{\ell^{\prime}}^{\beta}\right\}=-\delta_{\ell \ell^{\prime}} \sum_{\gamma} \epsilon^{\alpha \beta \gamma} S_{\ell}^{\gamma} .
$$

The classical 2-spin cluster model (1) represents an autonomous Hamiltonian system with two degrees of freedom and is therefore completely integrable if there exists an independent integral of the motion in addition to $H$. We have previously shown [3] that such an integral exists provided that the interaction parameters satisfy the relation

$$
\left(A_{x}-A_{y}\right)\left(A_{y}-A_{z}\right)\left(A_{z}-A_{x}\right)+\sum_{\alpha \beta \gamma=\operatorname{cycl}(x y z)} J_{\alpha}^{2}\left(A_{\beta}-A_{\gamma}\right)=0 .
$$

Autocorrelation functions for any component of one of the vectors $\mathbf{S}_{\ell}$ may be defined as averages over initial conditions (phase averages), or as averages over a trajectory that evolves from a single initial condition (time averages):

$$
\left\langle S_{\ell}^{\alpha}(t) S_{\ell}^{\alpha}(0)\right\rangle_{T}=\lim _{T \rightarrow \infty} \frac{1}{T} \int_{0}^{T} \mathrm{~d} t^{\prime} S_{\ell}^{\alpha}\left(t+t^{\prime}\right) S_{\ell}^{\alpha}\left(t^{\prime}\right)
$$


and

$$
\left\langle S_{\ell}^{\alpha}(t) S_{\ell}^{\alpha}(0)\right\rangle_{P}=(4 \pi)^{-2} \int \mathrm{d} \mathbf{S}_{1}(0) \int \mathrm{d} \mathbf{S}_{2}(0) S_{\ell}^{\alpha}(t) S_{\ell}^{\alpha}(0),
$$

where $\alpha=x, y, z$ and $\ell=1,2$. Here we concentrate on time averages. It has previously been suggested [6,7], that the long-time asymptotic behavior of the spin-autocorrelation function for chaotic trajectories is of the power-law type: $\sim t^{\beta-1}, \beta<1$. If that is the case, then the intensity spectrum, which is (as a consequence of the Wiener-Khinchin theorem) equal to the Fourier transform of the autocorrelation function, has a singularity $\sim \omega^{-\beta}$ as $\omega \rightarrow 0$. In previous work [8] we have already found power-law decay with $\beta<0$ of autocorrelation functions for particular chaotic trajectories of the 2 -spin model (1); in that case the intensity spectrum was non-divergent in the low-frequency limit. Here we report calculations for different trajectories of the same general model with very slow autocorrelation decay and consequent low-frequency divergence in the intensity spectrum. Figure 1 shows the (normalized) autocorrelation function $\left\langle S_{1}^{x}(t) S_{1}^{x}\right\rangle_{T} /\left\langle\left(S_{1}^{x}\right)^{2}\right\rangle_{T}$ up to time lag $t=200$ obtained via a time average over an interval of size $T=100000$ from the time series of a chaotic trajectory as specified in the caption. It exhibits a more or less uniform decay to zero. The inset to figure 1 shows the corresponding intensity spectrum as calculated from the autocorrelation function with time lag up to $t=8192$. It has an infrared power-law divergence with exponent $\beta \simeq 0.2$. This is consistent with the $\sim t^{-0.8}$ decay of the autocorrelation function observed at intermediate times.

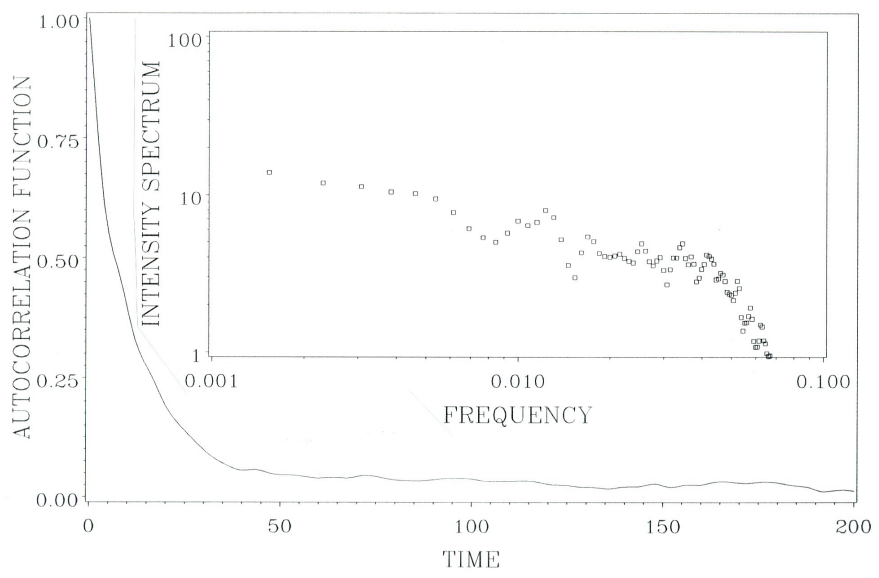

Figure 1. Normalized autocorrelation function $\left\langle S_{1}^{x}(t) S_{1}^{x}\right\rangle_{T} /\left\langle\left(S_{1}^{x}\right)^{2}\right\rangle_{T}$ and (inset) its Fourier transform for the (chaotic) trajectory which starts at $\theta_{1}=1.0, \theta_{2}=2.0, \phi_{1}=3.0, \phi_{2}=4.0$ of the nonintegrable classical 2-spin model specified by Hamiltonian (1) with parameter values $J_{x}=J_{y}=1, J_{z}=0, A_{x}=2, A_{y}=-1, A_{z}=0$.

Figure 2 shows the function $\left\langle S_{1}^{x}(t) S_{1}^{x}\right\rangle_{T} /\left\langle\left(S_{1}^{x}\right)^{2}\right\rangle_{T}$ and its intensity spectrum for a different chaotic trajectory and different model parameters (see caption for specifications). In this case, the autocorrelation function shows persistent high-frequency oscillations in addition to a slow powerlaw decay $\left(\sim t^{-0.6}\right)$. These features are reflected in the intensity spectrum by a power-law infrared divergence, $\sim \omega^{-0.4}$, and a sharp peak at $\omega \simeq 0.467$, respectively. A closer look (by stretching the frequency scale) reveals that this peak actually has some structure, which is dominated by two narrowly separated lines. They are the cause of the clearly distinguishable beats in the oscillations of the autocorrelation function shown in figure 2. These oscillations including the beats persist up to time lags of $t=4096$ without noticeable attenuation.

The structure of the correlation function can be understood in terms of trapping of the phase point in a small region of its allowed phase space, by nearly but not quite intact tori, as discussed for example by Hanson, Cary and Meiss [9] in the context of area preserving maps. Considering the effect of a single boundary torus, they concluded that decay $\sim t^{-2.05}$ would occur; considering 


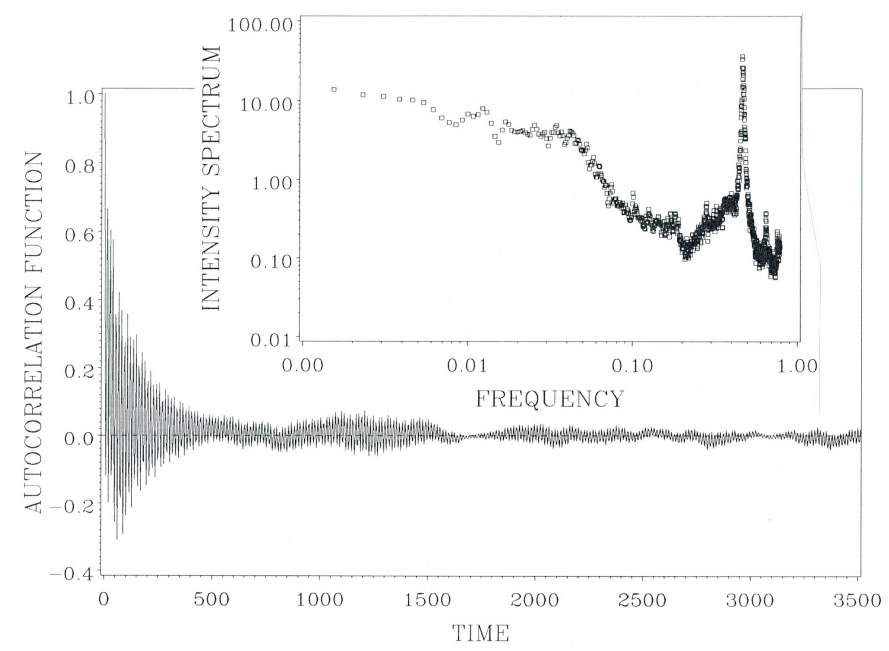

Figure 2. As in figure 1 , for the chaotic trajectory which starts at $\theta_{1}=0.471239, \theta_{2}=\pi / 2, \phi_{1}=$ $-\pi / 4, \phi_{2}=0.6988059$ and parameter values $J_{x}=J_{y}=1, J_{z}=0, A_{x}=-0.7, A_{y}=0.7, A_{z}=0$.

a hierarchy of tori, Meiss and Ott [10] find decay like $\sim t^{-0.96}$. While these theoretical predictions seem to accommodate our previous results (more rapid decay, $\sim t^{-1.5}$ ) [8] fairly well, the examples presented here involve considerably more slowly decaying time correlation functions, implying the existence of an even stronger trapping mechanism, which is yet to be understood.

Acknowledgment: This work was supported by the U.S. National Science Foundation Grant No. DMR-86-03036 and by a Grant-in-Aid of Research from Sigma Xi, The Scientific Research Society. We have used a modified cmpj.sty style file.

\section{References}

1. Feingold, M., Moiseyev, N., Peres, A., Phys. Rev. A 30 (1984) 509.

2. Frahm, H., Mikesa, H. J., Z. Phys. B 60 (1985) 117.

3. Magyari, E., Thomas, H., Weber, R, Kaufman, C., Müller, G., Z. Phys. B 65 (1987) 363.

4. Srivastava, N., Kaufman, C., Müller, G., Magyari, E., Weber, R, Thomas, H., J. Appl. Phys. 61 (1987) 4438.

5. Srivastava, N., Kaufman, C., Müller, G., Weber, R., Thomas, H., Z. Phys. B 70 (1988) 251.

6. Chirikov, B. V., Shepalyansky, D. L., Physica 13D (1984) 395.

7. Karney, C. F. F., Physica 8D (1983) 360.

8. Srivastava, N., Kaufman, C., Müller, G., J. Appl. Phys. 63 (1988) 4154.

9. Hanson, J. D., Cary, J. R, Meiss, J. D., J. Stat. Phys. 39 (1985) 327.

10. Meiss, J. D., Ott, E., Phys. Rev. Lett. 55 (1985) 2741; Physica 20D (1986) 387. 